\title{
3D registration of intravascular optical coherence tomography and cryo-image volumes for microscopic-resolution validation
}

\author{
David Prabhu $^{\mathrm{a}}$, Emile Mehanna ${ }^{\mathrm{b}}$, Madhusudhana Gargeshaa ${ }^{\mathrm{a}}$, Di Wen ${ }^{\mathrm{a}}$, Eric Brandt ${ }^{\mathrm{b}}$, Nienke \\ S. van Ditzhuijzen ${ }^{\mathrm{b}}$, Daniel Chamie ${ }^{\mathrm{b}}$, Hirosada Yamamoto $^{\mathrm{b}}$, Yusuke Fujino ${ }^{\mathrm{b}}$, Ali \\ Farmazilian $^{\mathrm{b}}$, Jaymin Patel ${ }^{\mathrm{a}}$, Marco Costa ${ }^{\mathrm{b}}$, Hiram G. Bezerra ${ }^{\mathrm{b}}$, and David L. Wilson ${ }^{\mathrm{a}}$ \\ aDepartment of Biomedical Engineering, Case Western Reserve University, Cleveland, Ohio \\ 44106 \\ bHarrington-McLaughlin Heart \& Vascular Institute, University Hospitals Case Medical Center \\ 'Department of Radiology, Case Western Reserve University, Cleveland, OH, 44106, USA
}

\section{Abstract}

High resolution, 100 frames/sec intravascular optical coherence tomography (IVOCT) can distinguish plaque types, but further validation is needed, especially for automated plaque characterization. We developed experimental and 3D registration methods, to provide validation of IVOCT pullback volumes using microscopic, brightfield and fluorescent cryo-image volumes, with optional, exactly registered cryo-histology. The innovation was a method to match an IVOCT pull-back images, acquired in the catheter reference frame, to a true 3D cryo-image volume. Briefly, an 11-parameter, polynomial virtual catheter was initialized within the cryo-image volume, and perpendicular images were extracted, mimicking IVOCT image acquisition. Virtual catheter parameters were optimized to maximize cryo and IVOCT lumen overlap. Local minima were possible, but when we started within reasonable ranges, every one of 24 digital phantom cases converged to a good solution with a registration error of only $+1.34 \pm 2.65 \mu \mathrm{m}$ (signed distance). Registration was applied to 10 ex-vivo cadaver coronary arteries (LADs), resulting in 10 registered cryo and IVOCT volumes yielding a total of 421 registered 2D-image pairs. Image overlays demonstrated high continuity between vascular and plaque features. Bland-Altman analysis comparing cryo and IVOCT lumen area, showed mean and standard deviation of differences as $0.01 \pm 0.43 \mathrm{~mm}^{2}$. DICE coefficients were $0.91 \pm 0$. 04 . Finally, visual assessment on 20 representative cases with easily identifiable features suggested registration accuracy within one frame of IVOCT $( \pm 200 \mu \mathrm{m})$, eliminating significant misinterpretations introduced by $1 \mathrm{~mm}$ errors in the literature. The method will provide 3D data for training of IVOCT plaque algorithms and can be used for validation of other intravascular imaging modalities.

\section{Keywords}

cryo-imaging; optical coherence tomography; 3D image registration; histology; intravascular imaging; validation

Address all correspondence to: David L Wilson, Case Western Reserve University, Biomedical Engineering, 10900 Euclid Ave, Cleveland, OH, 44106; Tel: 216-368-8812; dlw@ case.edu. 


\section{Introduction}

IVOCT has demonstrated great promise to identify key plaque features due to its resolution $(10-20 \mu \mathrm{m})$, noise, and contrast amongst rival intravascular imaging modalities (intravascular ultrasound, near infrared spectroscopy, intravascular MRI) $(1,2)$. IVOCT has been used to differentiate lipid, calcium, and fibrous tissue $(3,4)$ and can quantify macrophage content within atherosclerotic plaques (5). Furthermore, it is the only modality with the ability to quantify fibrous cap thickness: a powerful indicator of the likelihood of thin cap fibroatheroma (TCFA) rupture (6-8), which is believed to be the most frequent cause of myocardial infarction (MI) $(1,2,9,10)$.

However, challenges exist. Identification of a TCFA is not always straightforward. In particular, it is sometimes difficult to distinguish between lipid pools and large calcium deposits (1,11-13). Additionally, quantitative methods have been employed to automatically classify IVOCT images (14-17), but these are limited in scope as many are 2D, and many have insufficient data for machine learning approaches, which typically require a lot of data to cover the broad spectrum of vessel and lesion types an analyst may encounter. These challenges suggest a need for further validation studies of IVOCT imaging.

Many IVOCT validation studies currently use paraffin histology as the "gold standard" for validation $(4,5,18,19)$, but challenges exist with using this approach. First, sutures and ink marks, used to mark locations where histology sections are acquired, tend to be large as compared to IVOCT sampling (100 $\mu \mathrm{m}$ or $200 \mu \mathrm{m})$. Moreover, histological sections are often spatially distorted (tearing, folding, shearing, and shrinking) limiting the ability to make correlations even if the samples are in the same $z$ location $(18,20-22)$. Additionally, using these methods it would be very difficult to try to relate selected 2D histology images to the up to 500 images in a 3D IVOCT pullback.

Cryo-imaging is a novel imaging tool that should help fill the gap between 3D IVOCT pullbacks and 2D histology. Briefly, the system (CryoViz ${ }^{\mathrm{TM}}$, BioInVision) consists of a cryomicrotome with an integrated imaging system $(23,24)$. The system serially sections and acquires bright field and fluorescence microscopic image volumes. Visualization and analysis software is used to generate high resolution color and fluorescence volume renderings of vessels, in which plaque architecture and components are fully preserved. Furthermore, the system allows for individual sections to be collected and processed for standard histological staining.

In order to use cryo-imaging to routinely provide validation data for IVOCT images, we developed a specialized registration algorithm to match volumes of IVOCT images with corresponding cryo-image volumes. The major obstacle is that the cryo-image volume is a true 3D volume while the IVOCT volume is obtained from a pullback with the catheter tip as the reference for each image. We developed the algorithm and validated it on a synthetic digital phantom, as well as actual IVOCT and cryo-image data. We then show how this work can be applied toward developing automated IVOCT plaque characterization software. 


\section{Image Processing: Registration of Cryo-Image and IVOCT Pull-Back Volumes}

3D image registration software was created to match accurately a given IVOCT image with an appropriate image from the cryo-image volume. The challenge is that IVOCT images are obtained perpendicular to the imaging catheter with the imaging catheter always at the center of the image, while the cryo-image volume faithfully reflects the true 3D geometry and vessel shape at a much higher sampling rate.

Our solution was to digitally mimic the IVOCT acquisition process within the cryo-image volume. We created a virtual catheter within the cryo-image volume and extracted perpendicular, reformatted images. Since lumen segmentation is accurate in both modalities, we created an objective function based on the overlap of segmented binary images, and used normalized cross correlation to compute cost. This first step involved selecting volumes of interest (VOIs) from both the cryo and IVOCT images, and then segmenting the lumens in each dataset. We preformed registration on the segmented lumen volumes, and then applied the registration parameters to the full color cryo-data. The output was a volume of cryoimages that accurately matched the corresponding IVOCT images acquired from the same vessel.

We modelled the virtual catheter as a second order polynomial, governed by the equations below.

\section{Virtual Catheter Model}

$$
\begin{gathered}
\mathbf{X}_{\mathrm{c}}=\left(\frac{\alpha_{\mathrm{R}}}{\alpha_{\mathrm{z}}{ }^{2}} \cdot \mathrm{A}\right) \cdot\left(\boldsymbol{Z}_{c}\right)^{2}+\left(\frac{\alpha_{\mathrm{R}}}{\alpha_{\mathrm{z}}} \cdot \mathrm{B}\right)\left(\boldsymbol{Z}_{c}\right)+\alpha_{\mathrm{R}} \cdot \mathrm{C} \\
\boldsymbol{Y}_{\mathrm{c}}=\left(\frac{\alpha_{\mathrm{R}}}{\alpha_{\mathrm{z}}{ }^{2}} \cdot \mathrm{D}\right) \cdot\left(\boldsymbol{Z}_{c}\right)^{2}+\left(\frac{\alpha_{\mathrm{R}}}{\alpha_{\mathrm{z}}} \cdot \mathrm{E}\right)\left(\boldsymbol{Z}_{c}\right)+\alpha_{\mathrm{R}} \cdot \mathrm{F} \\
\boldsymbol{Z}_{\mathrm{c}}\left(\mathrm{k}_{\mathrm{c}}\right)=\left(\Delta \mathrm{z}_{\text {cryo }}\right) \cdot\left[\mathrm{k}_{\mathrm{c}}-\mathrm{k}_{0}\right]
\end{gathered}
$$

$[\mathrm{A}, \mathrm{B}, \mathrm{C}, \mathrm{D}, \mathrm{E}, \mathrm{F}]$ are coefficients of the polynomial terms; $\left[\mathrm{k}_{0}\right]$ is the starting index in the dataset; $\left[\mathrm{k}_{\mathrm{c}}\right]$ is the current index in the dataset; $\left[\mathrm{a}_{\mathrm{R}}\right]$ is the scaling factor along the radial direction; $\left[a_{\mathrm{Z}}\right]$ is the scaling factor along the $\mathrm{z}$ direction; $\left[\Delta \mathrm{z}_{\text {cryo }}\right]$ is the $\mathrm{z}$ resolution of the cryo-image volume.

To register the cryo and IVOCT volumes, we interactively select a point along the virtual catheter that corresponds to the first IVOCT image in a volume, using large side branches as fiducials. We then calculate the normal to the catheter at that point using the equations below. 


\section{Normal to Virtual Catheter}

$$
\begin{gathered}
\mathbf{X}_{\mathrm{c}}^{\prime}=2 \cdot\left(\frac{\alpha_{\mathrm{R}}}{\alpha_{\mathrm{z}}^{2}} \cdot \mathrm{A}\right) \cdot \boldsymbol{Z}_{c} \cdot \boldsymbol{Z}_{c}^{\prime}+\left(\frac{\alpha_{\mathrm{R}}}{\alpha_{\mathrm{z}}} \cdot \mathrm{B}\right) \cdot \boldsymbol{Z}_{c}^{\prime} \\
\boldsymbol{Y}_{\mathrm{c}}^{\prime}=2 \cdot\left(\frac{\alpha_{\mathrm{R}}}{\alpha_{\mathrm{z}}^{2}} \cdot \mathrm{D}\right) \cdot \boldsymbol{Z}_{c} \cdot \boldsymbol{Z}_{c}^{\prime}+\left(\frac{\alpha_{\mathrm{R}}}{\alpha_{\mathrm{z}}} \cdot \mathrm{E}\right) \cdot \boldsymbol{Z}_{c}^{\prime} \\
\boldsymbol{Z}_{\mathrm{c}}^{\prime}\left(\mathrm{z}_{\mathrm{c}}\right)=\Delta \mathrm{z}
\end{gathered}
$$

Using the point, $\mathbf{P}_{0}=\left(\mathbf{X}_{c}, \mathbf{Y}_{c}, \mathbf{Z}_{c}\right)$, and normal, $\mathbf{N}=\left\langle\mathbf{X}_{c}^{\prime}, \mathbf{Y}_{c}^{\prime}, \mathbf{Z}_{c}^{\prime}\right\rangle$, we can compute a plane within the cryo-volume, which corresponds to a particular IVOCT image. The equation for the plane is given below.

$$
\mathbf{X}_{c}^{\prime}\left(x-\mathbf{X}_{c}\right)+\boldsymbol{Y}_{c}^{\prime}\left(y-\boldsymbol{Y}_{c}+\boldsymbol{Z}_{c}^{\prime}\left(z-\boldsymbol{Z}_{c}\right)=0\right.
$$

$[x, y, z]$ is the position of each pixel in the IVOCT image plane. The pixel spacing of the plane is determined by the in-plane IVOCT resolution, $\Delta \mathrm{r}_{\mathrm{OCT}}$.

The plane is then rotated by an angle, $[\theta]$, to account for the rotation that takes place within the IVOCT dataset $(25,26)$. Each subsequent slice is then adjusted according to [d $\theta / \mathrm{dz}]$, which is the change in rotation that takes place as you move along the virtual catheter.

We then traverse a distance along the virtual catheter as determined by the $\mathrm{z}$ resolution of the IVOCT pullback, $\Delta \mathrm{z}_{\mathrm{OCT}}$, to find the starting point of the next image.

$$
\Delta \mathrm{z}_{\mathrm{OCT}}=\int_{a}^{b} \sqrt{\left[\mathbf{X}_{c}^{\prime}\right]^{2}+\left[\boldsymbol{Y}_{c}^{\prime}\right]^{2}+\left[\boldsymbol{Z}_{c}^{\prime}\right]^{2}}
$$

This process is repeated until each IVOCT image is matched with a corresponding cryoimage.

The catheter model was interactively initiated and iteratively refined using Nelder-Mead Simplex Optimization. Interactive initiation was done using a computer interface where parameters were manually adjusted by a user until a suitable starting condition was achieved. In total, each dataset involved optimizing the following 11 parameters, in order to find the best overlap between the IVOCT and Cryo images: $\Phi=\left[\mathrm{A}, \mathrm{B}, \mathrm{C}, \mathrm{D}, \mathrm{E}, \mathrm{F}, \mathrm{z}_{0}, \mathrm{a}_{\mathrm{R}}\right.$, $\left.a_{\mathrm{Z}}, \theta, \mathrm{d} \theta / \mathrm{dz}\right]$.

\section{Methods}

\subsection{Specimens and IVOCT Imaging}

We obtained coronary arteries from the Cuyahoga County Coroner's office from human cadavers within 72 hours of death. Arteries were removed and stored at $4{ }^{\circ} \mathrm{C}$ after removal 
from the body and prior to IVOCT imaging. The time between harvest and IVOCT imaging did not exceed one week. This procedure was deemed in accordance with federal, state, and local laws by the Case Institutional Review board.

Preparation of each artery was done prior to IVOCT imaging. A luer was sutured to the proximal end of each vessel. Vessels were flushed with saline to remove blood from the lumen. Major side branches and the distal end of each artery were sutured shut.

IVOCT imaging is now described. IVOCT image acquisition was performed using a stateof-the-art IVOCT imaging system, LightLab's C7-XR Fourier Domain IVOCT Intravascular Imaging System (C7-XR, LightLab Imaging Inc, MA). The recently FDA approved C7-XR is about $10 \times$ faster than the previous generation of time domain IVOCT. This provides a high density of A-lines per frame, which ultimately improves tissue features and consequently plaque characterization. The high sampling rate also enables faster pullbacks minimizing motion artifacts favoring $3 \mathrm{D}$ reconstructions of the vessel. Pullbacks were performed at 10.0 or $20.0 \mathrm{~mm} / \mathrm{s}$ using 50000 A-Lines per second yielding $5.4 \mathrm{~cm}$ pullbacks at 100 or 200 um frame intervals. Vessels were pressurized with saline or optimal cutting temperature compound (Tissue Tek, Ted Pella, Inc., Redding, CA) during the pullback to prevent vessel collapse. After IVOCT imaging, IVOCT images were reviewed and sutures were placed on the vessel corresponding to the start and stop points of regions of interest (ROI) along the vessel. Then, the imaging rig was filled with optimal cutting temperature compound. Following this the entire rig was covered in aluminum foil and snap frozen by placing it in a container filled with liquid nitrogen. The frozen specimen was then stored in a $-80{ }^{\circ} \mathrm{C}$ freezer until cryo-imaging was performed.

\subsection{Cryo-imaging and Acquisition of Corresponding Histology}

Preparation of each artery was done prior to cryo-imaging. Arteries were removed from the freezer, and cut into blocks corresponding to the ROI determined during IVOCT imaging. Prior to imaging, each block was placed in the cryo-imaging system to allow equilibration to the $-20{ }^{\circ} \mathrm{C}$ cutting temperature.

Cryo-imaging is now described. The cryo-imaging system consists of a modified large section cryo-microtome (8250 Large Section Cryostat, Vibratome, St. Louis, MO, U.S.A), $\mathrm{XYZ}$ robotic positioner carrying an imaging system which consists of a stereo microscope (SZX12, Olympus, Japan), GFP fluorescent filters (Exciter: HQ470/40×, Dichroic: Q495LP, Emitter: HQ500LP, Chroma, Rockingham, VT) low light digital camera (Retiga Exi, QImaging., Canada), color light source (XCite 120PC, EXFO, Canada). The cryo-imaging system is controlled by a control computer running Labview (National Instruments, Austin, TX). The stereo microscope used an objective of 0.11 NA and a zoom settings of 7-16x. Once equilibrated to cutting temperature, blocks were fixed to the stage of the cryomicrotome using optimal cutting temperature compound. The specimen was then sectioned until the surface of the block face was flat. The white light source, used for color imaging, was turned on and the camera was positioned over and focused onto the region of the block face surface containing the vessel. A white board was placed over the surface of the block face and was used to white balance the camera. The white board was removed and the bright-field exposure time for the camera was determined. For color imaging, typical 
exposure times for the green channel were $\sim 10 \mathrm{~ms}$. The white light was turned off, and the fluorescent light was turned on and the fluorescent exposure time was determined. For fluorescent imaging, typical exposure times for the green channel was $\sim 3000 \mathrm{~ms}$. The machine was then set to run such that a 20 um section was removed from the specimen, a bright-field followed by a fluorescence image of the block face was acquired, and the process was repeated until the whole specimen was imaged.

Histology was acquired at selected sections using the Kawamoto method (27). Histology slices were then digitized and registered to their corresponding cryo-image using $2 \mathrm{D}$ affine registration with: scale, rotation, and translation as free parameters.

\subsection{Image Processing Software}

Registration software was implemented in Matlab (Mathworks, Natick, MA). Initiation was done interactively by manually tweaking individual parameters until a suitable overlap between all lumen pairs was determined by visual assessment. The parameters were then iteratively refined using Nelder-Mead Simplex Optimization in Matlab, where cross correlation between lumen pairs was used as the cost function.

Registration of histology to corresponding cryo-images was done by digitizing histology slides using an Olympus VS120 Microscope. Digitized slides were then registered to their corresponding cryo-image using an affine transformation with: scale, rotation, and translation as free parameters. Mutual information was used as the cost function for cryo to histology registration.

All image processing software was implemented on a Dell Precision T7610 using a 64-bit edition of Windows 7 Professional, with two $2.60 \mathrm{GHz}$ Intel ${ }^{\circledR}$ Xeon ${ }^{\circledR}$ CPU with 128 GB of Ram.

\subsection{Assessment of Registration Accuracy}

We developed a digital phantom to validate the accuracy of our registration algorithm. To create the phantom, we used a binary lumen volume from a cryo-image dataset as a reference. We generated a sequence of 10 images from this volume using a known set of parameters with our virtual catheter model. These images were designated as "synthetic IVOCT images." Sets of initial parameter were chosen to include extremes of initiation errors that we thought reasonable, and mixtures of these errors. Additionally, we analyzed the effect of modifying the two stopping criteria: minimum change in parameter step size, and minimum change in the cost function. These stopping criteria were modified and their impact on registration accuracy was assessed. Because the images were digitally created, we knew the exact position of each voxel in the phantom images. Having this information, we were able to determine the Euclidean distance between the reference and our determined solution, as our measure of registration quality.

To determine registration quality on actual data, we conducted an experiment where we showed expert IVOCT image analysts an IVOCT image and 5 corresponding cryo images, which contained easily identifiable features, such as a side branch or a large well-defined calcified lesion. The five images contained the registered image as determined by our 
algorithm, two images before the registered image, and two images after the registered image. The images were spaced according to the frame interval of the IVOCT image $(200 \mu \mathrm{m})$. The analyst then selected the image that they felt matched best. The analyst was shown 20 such cases.

Additionally, we quantitatively assessed registration between IVOCT and cryo-image volumes by analyzing lumen area between registered images, as well as DICE coefficient of the corresponding lumens.

\subsection{Annotation of Registered Cryo-Images for Classifier Training}

We created a methodology for using cryo-imaging to prepare data for automated tissue classification studies. Using cryo images as a reference, an expert analyst marked every pixel in the IVOCT images that contained visible regions of: lumen, fibrous, lipid, or calcium. This database of annotated IVOCT images will be used as a ground truth repository for classifying IVOCT pullbacks in clinical settings.

\section{Results}

\subsection{Cryo-imaging/histology identification of major plaque types}

Cryo-imaging allows easy identification of common plaque types confronted in IVOCT imaging. In color and fluorescent cryo-images, one can determine features associated with normal, lipid, calcified, and fibrous plaque types, as shown in Figure 1. Calcified lesions (row 1) are characterized by a chalky white appearance in the color image, and high green signal in the fluorescent image. Lipid lesions (row 2) are characterized by a yellow appearance in the color image and a dim appearance in the fluorescence image. Fibrous tissue is seen (rows 1 and 2) in areas where the intimal layer of the vessel is inflamed and is characterized by a pink appearance in the color image, and a medium to bright appearance in the fluorescent image.

\subsection{Assessing registration quality with a digital synthetic phantom}

The digital phantom experiment assessed the registration accuracy of our algorithm (Methods 3.4). We evaluated the effect of potential local minima by starting the algorithm with different initial parameters and recording final registration results. Initial parameters were varied over ranges which would exceed the error in manually determined starting parameters. At these initial conditions, image transformations were clearly in error with image volume overlap as small as $10 \%$. Over twenty-four cases, the mean voxel error was $+1.3 \pm 2.7 \mu \mathrm{m}$, as determined by the signed Euclidean distance along $\mathrm{z}$, with the minimum (maximum) error being $+0.0 \pm 0.0 \mu \mathrm{m}(+7.2 \pm 3.6 \mu \mathrm{m})$.

\subsection{Assessing registration accuracy of IVOCT to cryo-volumes}

We assessed the quality of registration for actual IVOCT and cryo-image volumes. In a sequence of registered images, we compared the frames before and after the corresponding image (Fig. 2). We did this for twenty cases, and found that for the majority of cases, each frame matched its corresponding image better than the frame before and the frame after. This suggests that the accuracy of our registration is within 1 frame interval $( \pm 200 \mu \mathrm{m})$. 
We applied additional quantitative metrics to assess registration of IVOCT and cryo image data. We compare lumen areas and DICE coefficients for corresponding frames in each registered volume (Fig. 3). A clear correlation is observed in both metrics. Bland-Altman analysis (3a) comparing cryo and IVOCT lumen area, showed 401 of 421 cases within the $95 \%$ confidence interval, with mean and standard deviation of the differences being 0.01 and $0.43 \mathrm{~mm}^{2}$, respectively. Mean DICE coefficients (3b) for all cases were $0.91 \pm 0.04$.

\subsection{Application to plaque characterization}

Our work can be used to provide annotated training and testing data for plaque characterization algorithms (Fig. 4). Although registration is quite accurate, we choose to use cryo-images as a guide to annotate IVOCT images. Due to potential, small registration errors, this is more accurate than annotating the cryo-images and copying results directly to IVOCT. In this way, we create annotated IVOCT images, providing an accurate training set for machine learning algorithms. In Fig. 8, calcium and lipid regions are labeled and easily identified in cryo-images and IVOCT images.

\section{Discussion}

\subsection{D validation of IVOCT images achieved with high registration accuracy}

In this study, we developed a methodology to provide accurate 3D validation of IVOCT pullback volumes using microscopic, color and fluorescent cryo-image volumes. We achieved clear identification of plaque types using cryo-imaging, with very accurate registration to individual IVOCT frames, enabling one to find a feature in cryo-images and look for the corresponding response in IVOCT. Potential applications at least include: verification of IVOCT manual classification, investigations of plaque pathobiology, creation of an IVOCT atlas or physician training data, and creation of databases for machine learning plaque classification algorithms.

For cryo-IVOCT registration, we created a specialized image registration method. The issue is that IVOCT is obtained with the catheter tip as the image reference, while cryo-imaging reveals the true shape of the vessel. Our solution consisted of creating a virtual catheter path through the cryo-image volume. At least over the segment lengths studied here, a $2^{\text {nd }}$ order polynomial was sufficient for modeling the path. We used binary lumen volume overlap as the objective function. We found that there were sufficient features (side branches and nonconcentric lumens) that lumen volume worked well.

Registration accuracy is better than the spacing between IVOCT image frames $(200 \mu \mathrm{m})$. Errors estimated with the digital synthetic phantom were very small $(+1.3 \pm 2.7 \mu \mathrm{m}$ in $\mathrm{z})$. When operators evaluated registration accuracy on real data (Methods 3.4), accuracy appeared to be within one IVOCT frame interval. DICE and lumen area metrics (Fig. 3a and 3b) matched very well. Small displacement errors were likely due to small distortions of the vessels between IVOCT imaging and cryo-imaging procedures. Rather than continuing the registration process, possibly with additional deform-able registration, we chose another strategy. We go to great lengths to register the appropriate cryo-image to the corresponding IVOCT image. We then allow the operator to annotate the IVOCT image using the registered 
cryo-image as a guide. There is little or no ambiguity in annotation. Our proposed method should limit the misinterpretations which are clearly possible with other methods.

Furthermore, the added benefit of having 3D data allows one to analyze adjacent regions to individual IVOCT frames as well, effectively allowing one to circumvent the effects any registration error may have on plaque characterization.

We believe that our method will be ideal for creating training and testing data for automated classification (Fig. 8). Each tissue voxel in an IVOCT volume can be labeled, allowing even voxel-based classification rather than region based classification. Because we can label each image frame in a pullback sequence, we can utilize 3D information in classification. This would not be possible with isolated histology images. Finally, our methods can be used to validate other intravascular imaging modalities as well, including: intravascular MRI, intravascular ultrasound, near infrared fluorescence, and multimodality solutions which combine these modalities. Each of these methods employs a catheter pull-back in image acquisition, which can be handled using our methodology.

\subsection{Conclusion}

We believe that cryo-imaging is a promising technology for validation of 3D IVOCT imaging studies. It enables heretofore unavailable registration accuracy between IVOCT imaging data and 3D microscopic cryo-image data with optional histology. The method will provide 3D data for training of IVOCT plaque classification algorithms. The virtual catheter registration method and cryo-imaging should be applicable for assessments of other intravascular imaging modalities.

\section{Acknowledgments}

This project was supported by the National Heart, Lung, and Blood Institute through grants NIH R21HL108263 and 1R01HL114406-01, the Choose Ohio First Scholarship, and by the National Center for Research Resources and the National Center for Advancing Translational Sciences through grant UL1RR024989. These grants were attained via collaboration between Case Western Reserve University and University Hospitals of Cleveland.

\section{References}

1. Virmani R, et al. Lessons from sudden coronary death: a comprehensive morphological classification scheme for atherosclerotic lesions. Arteriosclerosis, thrombosis, and vascular biology. 2000; 20(5):1262-1275.

2. Lee RT, Libby P. The unstable atheroma. Arteriosclerosis, thrombosis, and vascular biology. 1997; 17(10):1859-1867.

3. Suh WM, et al. Intravascular detection of the vulnerable plaque. Circulation Cardiovascular imaging. 2011; 4(2):169-178. [PubMed: 21406663]

4. Puri R, Worthley MI, Nicholls SJ. Intravascular imaging of vulnerable coronary plaque: current and future concepts. Nature reviews Cardiology. 2011; 8(3):131-139. [PubMed: 21263456]

5. Yabushita H, et al. Characterization of human atherosclerosis by optical coherence tomography. Circulation. 2002; 106(13):1640-1645. [PubMed: 12270856]

6. Kume T, et al. Measurement of the thickness of the fibrous cap by optical coherence tomography. American heart journal. 2006; 152(4):755 e751-754. [PubMed: 16996853]

7. Chamie D, et al. Optical Coherence Tomography and Fibrous Cap Characterization. Current cardiovascular imaging reports. 2011; 4(4):276-283. [PubMed: 21949565]

8. Wang $\mathrm{Z}$, et al. Volumetric quantification of fibrous caps using intravascular optical coherence tomography. Biomedical optics express. 2012; 3(6):1413-1426. [PubMed: 22741086] 
9. Burke AP, et al. Coronary risk factors and plaque morphology in men with coronary disease who died suddenly. The New England journal of medicine. 1997; 336(18):1276-1282. [PubMed: 9113930]

10. Virmani R, et al. Pathology of the vulnerable plaque. Journal of the American College of Cardiology. 2006; 47(8 Suppl):C13-18. [PubMed: 16631505]

11. Kang SJ, et al. Optical coherence tomographic analysis of in-stent neoatherosclerosis after drugeluting stent implantation. Circulation. 2011; 123(25):2954-2963. [PubMed: 21646494]

12. Tearney GJ, et al. Quantification of macrophage content in atherosclerotic plaques by optical coherence tomography. Circulation. 2003; 107(1):113-119. [PubMed: 12515752]

13. Jang IK, et al. Visualization of coronary atherosclerotic plaques in patients using optical coherence tomography: comparison with intravascular ultrasound. Journal of the American College of Cardiology. 2002; 39(4):604-609. [PubMed: 11849858]

14. van Soest G, et al. Atherosclerotic tissue characterization in vivo by optical coherence tomography attenuation imaging. Journal of biomedical optics. 2010; 15(1):011105. [PubMed: 20210431]

15. Ughi GJ, et al. Automated tissue characterization of in vivo atherosclerotic plaques by intravascular optical coherence tomography images. Biomedical optics express. 2013; 4(7):1014-1030. [PubMed: 23847728]

16. $\mathrm{Xu} \mathrm{C}$, et al. Characterization of atherosclerosis plaques by measuring both backscattering and attenuation coefficients in optical coherence tomography. Journal of biomedical optics. 2008; 13(3):034003. [PubMed: 18601548]

17. Gargesha M, et al. Parameter estimation of atherosclerotic tissue optical properties from threedimensional intravascular optical coherence tomography. Journal of medical imaging. 2015; 2(1): 016001. [PubMed: 26158087]

18. van Soest G, et al. Pitfalls in plaque characterization by OCT: image artifacts in native coronary arteries. JACC Cardiovascular imaging. 2011; 4(7):810-813. [PubMed: 21757174]

19. Kubo T, et al. Virtual histology intravascular ultrasound compared with optical coherence tomography for identification of thin-cap fibroatheroma. International heart journal. 2011; 52(3): 175-179. [PubMed: 21646741]

20. Kume T, et al. Assessment of coronary arterial plaque by optical coherence tomography. The American journal of cardiology. 2006; 97(8):1172-1175. [PubMed: 16616021]

21. Virmani R, et al. Effect of aging on aortic morphology in populations with high and low prevalence of hypertension and atherosclerosis. Comparison between occidental and Chinese communities. The American journal of pathology. 1991; 139(5):1119-1129. [PubMed: 1951629]

22. Siegel RJ, et al. Limitations of postmortem assessment of human coronary artery size and luminal narrowing: differential effects of tissue fixation and processing on vessels with different degrees of atherosclerosis. Journal of the American College of Cardiology. 1985; 5(2 Pt 1):342-346. [PubMed: 3881498]

23. Roy D, et al. 3D cryo-imaging: a very high-resolution view of the whole mouse. Anatomical record. 2009; 292(3):342-351.

24. Roy D, et al. Imaging System for Creating 3D Block-Face Cryo-Images Of Whole Mice. Proceedings of SPIE--the International Society for Optical Engineering. 2006; 6143:nihpa1 12282. [PubMed: 19802364]

25. Ahsen OO, et al. Correction of rotational distortion for catheter-based en face OCT and OCT angiography. Optics letters. 2014; 39(20):5973-5976. [PubMed: 25361133]

26. van Soest G, Bosch JG, van der Steen AF. Azimuthal registration of image sequences affected by nonuniform rotation distortion. IEEE transactions on information technology in biomedicine : a publication of the IEEE Engineering in Medicine and Biology Society. 2008; 12(3):348-355.

27. Kawamoto T, Shimizu M. A method for preparing 2- to 50-micron-thick fresh-frozen sections of large samples and undecalcified hard tissues. Histochemistry and cell biology. 2000; 113(5):331339. [PubMed: 10883392]

Proc SPIE Int Soc Opt Eng. Author manuscript; available in PMC 2016 May 07. 



Figure 1. Major plaque tissue types are recognizable in cryo-images

Cryo-imaging easily distinguishes the major plaque types (calcium, fibrous, and lipid) confronted during IVOCT imaging. Calcified lesions (row 1) are characterized by a chalky white appearance in the color image, and high green signal in the fluorescent image. Lipid lesions (row 2) are characterized by a yellow appearance in the color image and a dim appearance in the fluorescence image. Fibrous tissue is seen (rows 1 and 2) in areas where the intimal layer of the vessel is inflamed, and is characterized by a pink appearance in the color image, and a medium to bright appearance in the fluorescent image. There are large regions of fibrous tissue, and $\mathrm{F}$ identifies a classic appearance. 


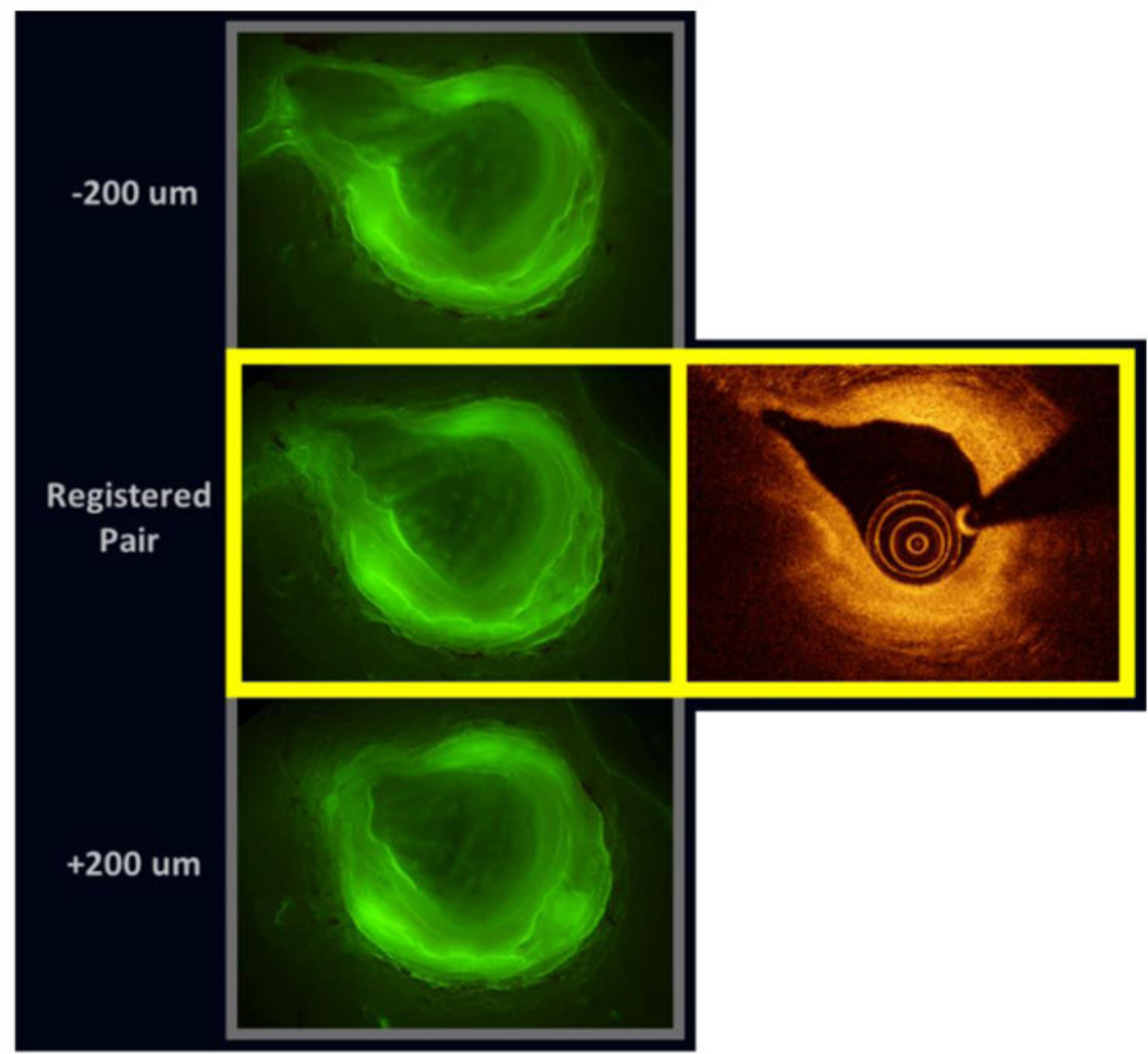

Figure 2. Qualitative analysis of registered cryo and IVOCT image datasets, suggests zregistration accuracy is within 1 IVOCT frame interval $( \pm 200 \mu \mathrm{m})$

We visually compared registered cryo- and IVOCT image pairs, as well as images located immediately prior and post within the registered datasets. Qualitative features suggested that registration accuracy was within 1 frame interval. 


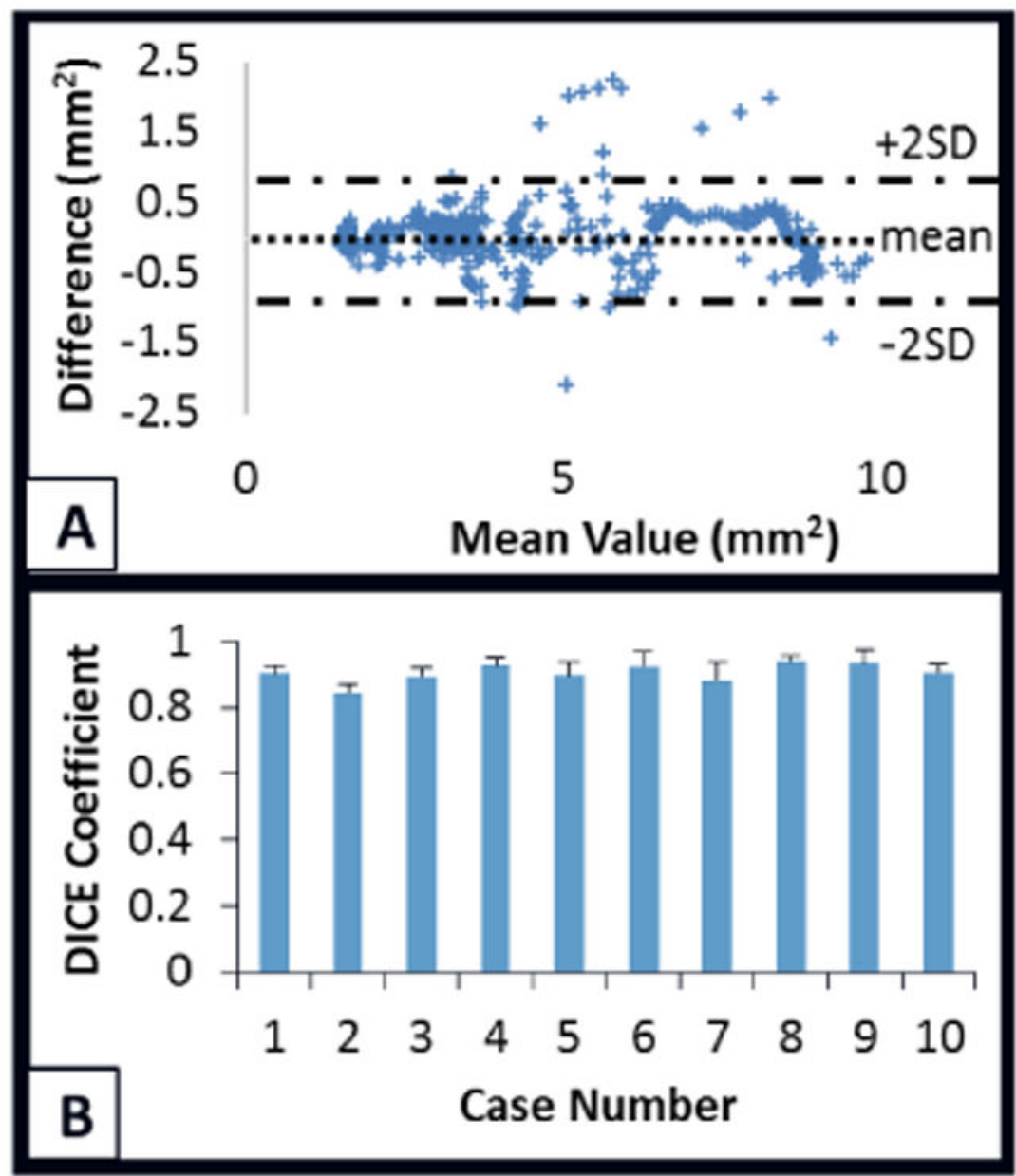

Figure 3. Qualitative lumen metrics again suggest very high registration between cryo and IVOCT image pairs

In (a), Bland-Altman analysis compares the lumen areas for all 441 registered pairs generated in the study. We see 421 of 441 cases within the $95 \%$ confidence interval of agreement, with mean and standard deviation of the differences being 0.01 and $0.43 \mathrm{~mm}^{2}$. Mean DICE for each case is shown in (b), with standard deviation shown in the error bars. Mean DICE for all cases were $0.91 \pm 0.04$. Both metrics suggest very high registration accuracy. 



Figure 4. Registered cryo-images can be used to train and test automated plaque characterization methods for IVOCT images

Using the registered cryo-image as reference, we can annotate every pixel in an IVOCT image, corresponding to a major plaque type (fibrous, lipid, calcium). Using these annotated images, we can test and train a classifier to automatically characterize IVOCT images. In panels A-C, we show a case containing a lipid lesion, denoted by the letter ' $\mathrm{L}$ '. Fibrous tissue is shown as a gray ring. 